Review began 12/30/2021 Review ended 01/13/2022 Published 01/13/2022

๑) Copyright 2022

Alsharif et al. This is an open access article distributed under the terms of the Creative Commons Attribution License CC-BY 4.0. which permits unrestricted use, distribution, and reproduction in any medium, provided the original author and source are credited.

\section{Assessment of the Response to Abdominal and Pelvic Computed Tomography Report Recommendations: A Single-Center, Retrospective, Chart Review Study}

Shaza Alsharif 1, 2, 3 , Ghalib Alasaad ${ }^{2}$, Mohammed K. Bukhari ${ }^{4,1}$, Abdulaziz Sharkar ${ }^{2}$, Mohammed Altaf ${ }^{2}$ , Shaymaa Milibari ${ }^{5}$, Roaa Alsolaimani ${ }^{6}$, Khalid M. Alshamrani $1,3,7$

1. Research, King Abdullah International Medical Research Center, Jeddah, SAU 2. College of Medicine, King Saud Bin Abdulaziz University for Health Sciences, Jeddah, SAU 3. Medical Imaging, Ministry of National Guard - Health Affairs, Jeddah, SAU 4. Neurology, College of Medicine, King Saud Bin Abdulaziz University for Health Sciences, Jeddah, SAU 5. Department of Quality, King Faisal Residential City Clinic, Jeddah, SAU 6. College of Medicine, King Abdulaziz University, Jeddah, SAU 7. College of Applied Medical Sciences, King Saud Bin Abdulaziz University for Health Sciences, Jeddah, SAU

Corresponding author: Shaza Alsharif, dr.salsharif@gmail.com

\section{Abstract \\ Objectives}

The radiology report is the primary form of communication between the radiologists and referring clinicians. It is a structured document containing several key components pertaining to the interpretation of radiological examinations and may require the addition of follow-up imaging recommendations to optimize patient outcomes. This study aims to determine whether follow-up imaging recommendations are being acknowledged and acted upon by referrers.

\section{Methods}

This retrospective study was conducted at a single tertiary hospital. Prerecorded BESTCare data of patients who underwent abdominal and pelvic computed tomography (CT) scans between October 1, 2017, and December 31, 2017, and received recommendations for further evaluation were collected after obtaining ethical approval from the local authority. Data of patients younger than 14 years old, patients who did not receive a recommendation, and patients who had CT scans that were uploaded to the BESTCare system but were performed outside the institution were excluded. The collected data were recorded in a passwordprotected Microsoft Excel file for further analysis.

\section{Results}

A total of 523 report recommendations from 422 abdominal and pelvic CT reports were analyzed. The most common organs indicated for CT scan evaluation were the breast ( $\mathrm{N}=54,10.33 \%)$, kidney ( $\mathrm{N}=46,8.80 \%$ ), lymph node $(\mathrm{N}=36,6.88 \%)$, and colon $(\mathrm{N}=33,6.31 \%)$. The most common type of further evaluation recommended was further imaging $(\mathrm{N}=410,78.39 \%)$. A total of $278(53.15 \%)$ recommendations were not performed, with 199 (71.58\%) not having a documented rationale for noncompliance.

\section{Conclusion}

The majority of the follow-up imaging recommendations to ordering physicians were not carried out. This study highlights the need for notification and audit systems to monitor compliance with follow-up recommendations. Improving the communication between radiologists and referring physicians is key to optimizing patient healthcare.

Categories: Radiology, Quality Improvement, Health Policy

Keywords: abdominal and pelvic computed tomography, medical imaging informatics, health informatics, compliance, follow-up recommendations, ct scan reports, radiologist recommendation, radiology report

\section{Introduction}

The radiology report is a structured document containing several key components pertaining to the interpretation of radiological examinations that can be used to narrow the differential diagnosis and may require the addition of follow-up imaging recommendations to optimize patient outcomes and care [1,2]. It is the primary form of communication between radiologists and referring physicians toward integrating clinical information with the radiological findings and data from other departments [3-5]. The need for effective communication has greatly increased owing to the existence of a wide spectrum of medical subspecializations, the advancement of medical technologies, and the significance of findings from many 
departments to reach a diagnosis and commence management [6,7]. Furthermore, the increased complexity of recent medical imaging techniques stresses the need for accurate presentation and description of findings [5]. Radiology reports may require the addition of recommendations that could assist in clearing up any uncertainties, confirming a diagnosis, or reporting incidental findings, and therefore, such recommendations may greatly impact the healthcare services delivered to the patient [3]. A study conducted in Waikato Hospital in Hamilton, New Zealand, reported that the likelihood of further investigation of adrenal incidentalomas according to guidelines increased as a result of radiology report recommendations [8]. It is expected that these recommendations will be acknowledged by the referring physicians, as any disregard for radiology report recommendations may negatively impact the quality of the service. Low adherence to recommendations regarding follow-up imaging may lead to subsequent poor healthcare delivery, avoidable unnecessary tests, and liability for legal action $[9,10]$.

Prior studies have discussed the response to radiology report recommendations in various fields and the rate at which the recommendations were carried out. However, to the best of our knowledge, there are no former studies specifically directed toward the response to abdominal and pelvic computed tomography (CT) report recommendations. The aim of this retrospective study is to assess referrers' responses to follow-up radiology report recommendations. We further highlight the potential reasons for the variation in the response to recommendations with the goal of improving communication between referring physicians and radiologists, which will lead to an improvement in the quality of service delivered to patients.

\section{Materials And Methods \\ Study design and sampling technique}

This study implemented a retrospective, chart review study design and a non-probability convenient sampling technique to collect the radiology reports of all abdominal and pelvic CT performed between October 1, 2017, and December 31, 2017, in King Abdulaziz Medical City, Ministry of National Guard Health Affairs (MNG-HA), Jeddah City, Kingdom of Saudi Arabia (KSA).

\section{Inclusion and exclusion criteria}

The subjects included in this research were all adults who underwent abdominal and pelvic CT and received a recommendation for further evaluation. Patients under 14 years of age, patients who did not receive a recommendation, and patients who had CT scans that were uploaded to the BESTCare system but were performed outside the institution were excluded.

\section{Data collection}

Access to patient documents and electronic files was through the electronic medical record system (BESTCare). The collection of the data was self-administered by four medical students into a passwordprotected Microsoft Excel sheet after obtaining institutional review board (IRB) approval from the local authority. Patients were categorized according to age, gender, the type of recommendation received, the referral department and indication for the CT scan, whether the recommendation was in the same organ system imaged, and whether the recommendation was done or not.

\section{Ethical consideration}

The ethics committee of King Abdullah International Medical Research Center issued institutional review board (IRB) approval \#SP18/331/J. The data were anonymized, and confidentiality of patient information was maintained throughout the project.

\section{Statistical analysis}

The data were analyzed using the statistical package IBM SPSS version 24 and Minitab version 17. Initial descriptive analysis (i.e., mean and standard deviation and percentages) of demographical characteristics was generated. The Chi-square test of independence was used to determine the relationship between a) the recommendations that were done and whether they were done in the same system as the CT indication and b) the recommendations that were done and the type of further evaluation recommended. A p-value of less than 0.05 was considered significant.

\section{Results}

A total number of 423 abdominal and pelvic CT reports were included in this study from 413 patients. The reports contained a total of 550 recommendations, of which 523 recommendations were analyzed. The remaining 27 recommendations were excluded due to missing information. The patients' ages range from 14 to 103 years (mean $\pm \mathrm{SD}=55.14 \pm 17.39$ ), $244(59 \%)$ of which are females and $169(41 \%)$ are males. Patients undergoing abdominal and pelvic CT were referred from different departments. The majority of reports with radiology recommendations were referred from the Adult Medical Oncology Department (124/413, 30.02\%) and the Emergency Department (76/413, 18.40\%), followed by the Gastroenterology Department (31/413, $7.51 \%$ ) and the General Surgery Department (30/413, 7.26\%) (Table 1). 


\section{Cureus}

\section{Demographic Variables}

Age $($ mean \pm SD $)$

Gender

Male

Female

Referral Department

Adult Medical Oncology

Emergency Medicine

Gastroenterology

General Surgery

Internal Medicine

Urology

Radiation Oncology

Neurology

Thoracic Surgery

Others
Frequency $(\mathrm{N}=413)$

Percentage

$55.14 \pm 17.39$

169

244

$41 \%$

$59 \%$

$30.02 \%$

$18.40 \%$

$7.51 \%$

$7.26 \%$

$5.57 \%$

$5.57 \%$

$3.63 \%$

$3.39 \%$

$2.42 \%$

$16.22 \%$

\section{TABLE 1: Patient Demographics}

The only exceptions are that the General Surgery Department received more recommendations than the Gastroenterology Department and the Neurology Department received more recommendations than the Radiation Oncology Department (Figure 1, Table 2).

\begin{tabular}{|l|l|l|}
\hline Row Labels & Number of Recommendations Received by the Referring Departments & Percentage \\
\hline Adult Medical Oncology & 155 & $29.64 \%$ \\
\hline Emergency Medicine & 79 & $15.11 \%$ \\
\hline General Surgery & 41 & $7.84 \%$ \\
Gastroenterology & 35 & $6.69 \%$ \\
\hline Internal Medicine & 35 & $6.69 \%$ \\
\hline Urology Surgery & 25 & $4.78 \%$ \\
\hline Neurology & 16 & $4.21 \%$ \\
\hline Radiation Oncology & 15 & $3.06 \%$ \\
\hline Thoracic Surgery & 22 & $2.87 \%$ \\
\hline
\end{tabular}

TABLE 2: Recommendations Received by the Referring Departments 


\section{Cureus}

Row Labels

Breast

Kidney

Lymph node

Colon

Liver

Bowel

Lungs

Abdomen

Malignancy

Abdominal wall

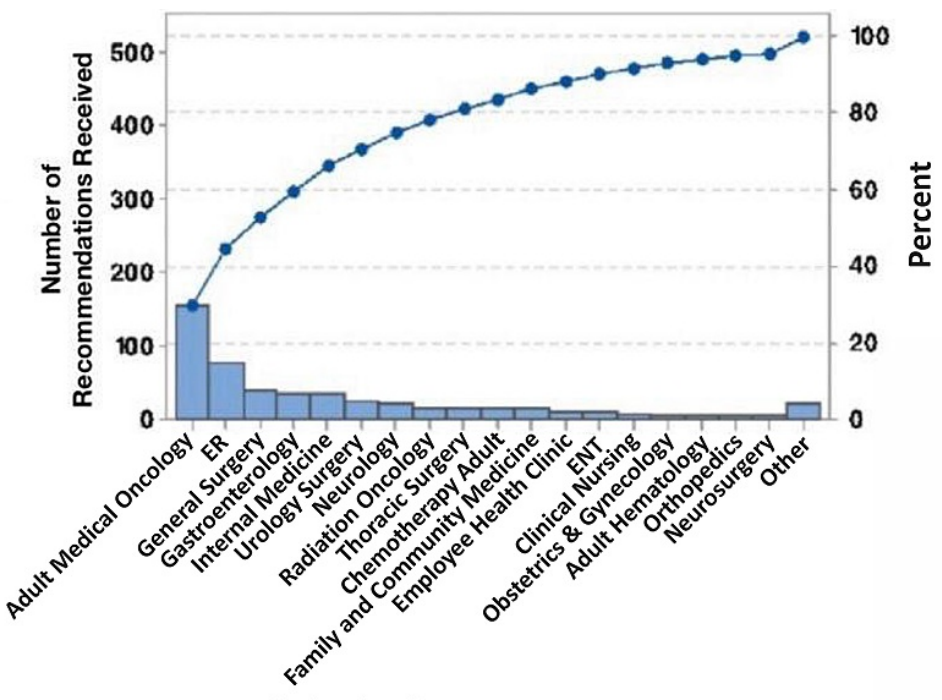

Referring Department

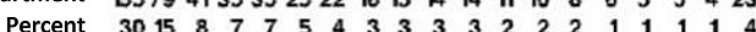

Cum \% 304553596671757881848688909293949596100

FIGURE 1: Recommendations Received by the Referring Departments

The patients whose recommendations were analyzed (some of whom received more than one recommendation) underwent a CT scan due to various medical indications. The most common organs indicated for CT scans were the breast (54/423, 12.77\%), kidney (46/423, 10.87\%), lymph node (36/423, $8.51 \%)$, and colon $(33 / 423,7.80 \%$ ) (Table 3).

TABLE 3: Indication for Abdominal and Pelvic CT

Sorted into different categories, the most common types of further evaluation recommended were further imaging (410/523, 78.39\%), further laboratory evaluation (60/523, 11.47\%), non-imaging procedures (37/523, $7.07 \%$ ), and specialty referrals (16/523, 3.06\%) (Figure 2, Table 4). 


\section{Cureus}

\begin{tabular}{|c|c|c|}
\hline Row Labels & Type of Recommendation Received & Percentage \\
\hline Imaging & 410 & $78.39 \%$ \\
\hline Laboratory & 60 & $11.47 \%$ \\
\hline Non-imaging procedure & 37 & $7.07 \%$ \\
\hline Specialty referral & 16 & $3.06 \%$ \\
\hline Grand total & 523 & $100 \%$ \\
\hline
\end{tabular}

TABLE 4: Type of Further Evaluation Recommended

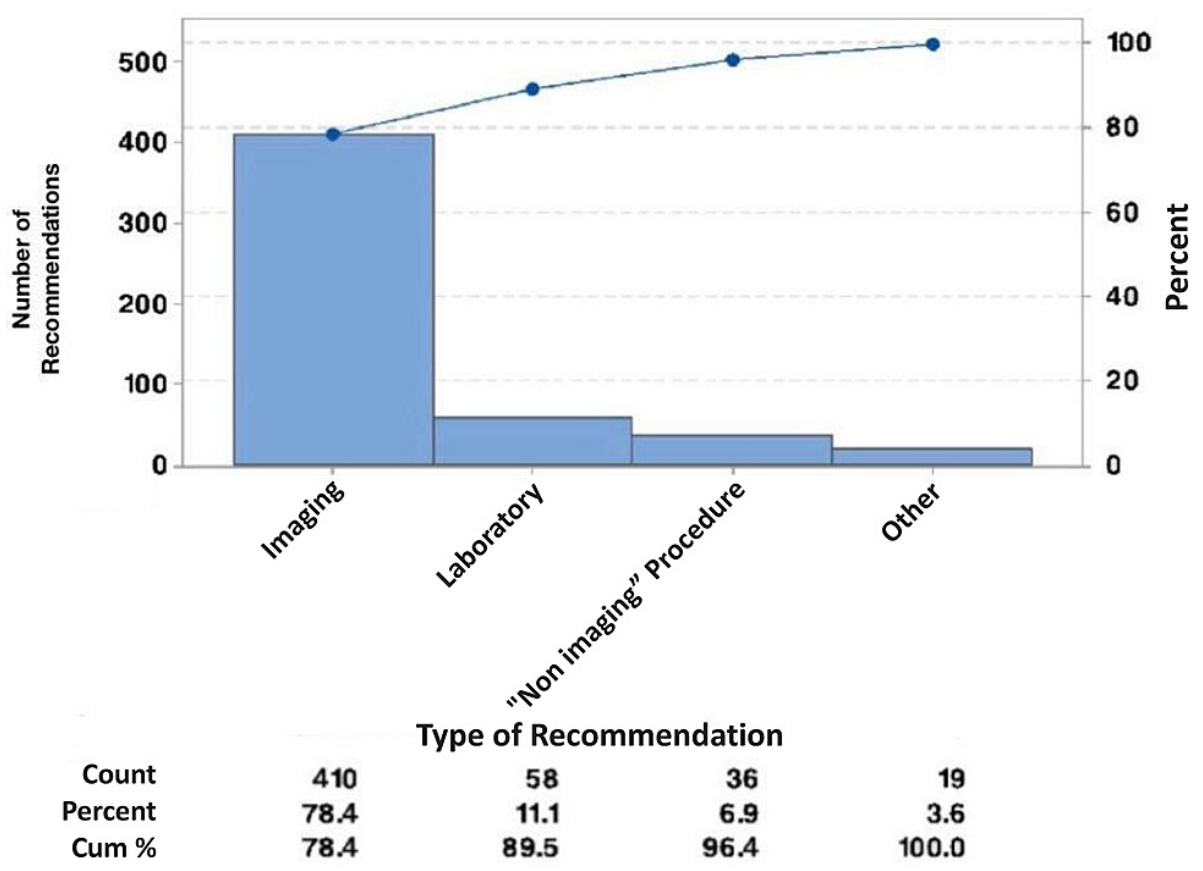

FIGURE 2: Type of Further Evaluation Recommended

The renal organ system received the most recommendations for further evaluation (111/523, 21.23\%), followed by the hepatobiliary system $(101 / 523,19.31 \%)$ and the genitourinary system $(88 / 523,16.83 \%)$ (Table 5). 


\section{Cureus}

\begin{tabular}{|c|c|c|}
\hline Organ System Examined & Number of Organ System Examined & Percentage \\
\hline Renal & 111 & $21.23 \%$ \\
\hline Hepatobiliary & 101 & $19.31 \%$ \\
\hline Genital & 88 & $16.83 \%$ \\
\hline Other & 63 & $12.04 \%$ \\
\hline Gastrointestinal & 50 & $9.56 \%$ \\
\hline Respiratory & 32 & $6.12 \%$ \\
\hline Musculoskeletal & 42 & $8.03 \%$ \\
\hline Lymphatic system & 22 & $4.21 \%$ \\
\hline Pulmonary & 13 & $2.49 \%$ \\
\hline Cardiovascular & 1 & $0.19 \%$ \\
\hline Grand total & 523 & $100 \%$ \\
\hline
\end{tabular}

\section{TABLE 5: Organ System Recommended to be Evaluated Further}

There were 158/523 (30.21\%) recommendations in the same organ system as the indication for the abdominal and pelvic CT, whereas 358/523 (68.45\%) of the recommendations were not in the same organ system, and 7/523 (1.34\%) of the recommendations were not applicable to the criteria (Table 6 ).

\begin{tabular}{|l|l|l|}
\hline Is the Recommendation in the Same Organ System as the CT Indication? & Number & Percentage \\
\hline No & 358 & $68.45 \%$ \\
\hline Yes & 158 & $30.21 \%$ \\
\hline Inconclusive & 7 & $1.34 \%$ \\
\hline Grand total & 523 & $100 \%$ \\
\hline
\end{tabular}

TABLE 6: Relationship of Organ System Recommended to be Evaluated Further to the CT Indication

Of the 523 recommendations studied, 278 (53.15\%) were not performed and 245 (46.85\%) of the recommendations were performed. The recommendations performed within six months were $202(38.62 \%)$, and 321 (61.38\%) were not performed within six months (Table 7).

\begin{tabular}{|l|l|l|}
\hline Done Within Six Months? & Number & Percentage \\
\hline No & 321 & $61.38 \%$ \\
\hline Yes & 202 & $38.62 \%$ \\
\hline Grand total & 523 & $100 \%$ \\
\hline
\end{tabular}

TABLE 7: Number of Recommendations Performed and Not Performed Within Six Months

The number of non-performed recommendations was 278 (53.15\%), whereas the number of performed recommendations was 245 (46.85\%), of which 43 (17.55\%) recommendations were not performed within a six-month period (Table 8 ). 


\section{Cureus}

\begin{tabular}{|c|c|c|}
\hline Recommendation Done? & Number & Percentage \\
\hline No & 278 & $53.15 \%$ \\
\hline Yes & 245 & $46.85 \%$ \\
\hline Grand total & 523 & $100 \%$ \\
\hline
\end{tabular}

TABLE 8: Number of Recommendations Performed

It should be noted that some recommendations were for follow-up of certain findings after a six-month period. Of the 278 non-performed recommendations, 199 (71.58\%) did not have a documented rationale, and 58 (20.86\%) were not performed because an alternative study was done. Additionally, 12 (4.32\%) were not performed because a study was already performed before the CT scan (Figure 3, Table 9).

\begin{tabular}{|c|c|c|}
\hline Reason for Not Performing the Recommendation & Number & Percentage \\
\hline No documentation of rationale & 199 & $71.58 \%$ \\
\hline Alternative study done & 58 & $20.86 \%$ \\
\hline Done prior to examination & 12 & $4.32 \%$ \\
\hline Patient no show & 8 & $2.88 \%$ \\
\hline Appointment postponed & 1 & $0.36 \%$ \\
\hline Grand total & 278 & $100 \%$ \\
\hline
\end{tabular}

TABLE 9: Rationale for Not Performing the Recommendation

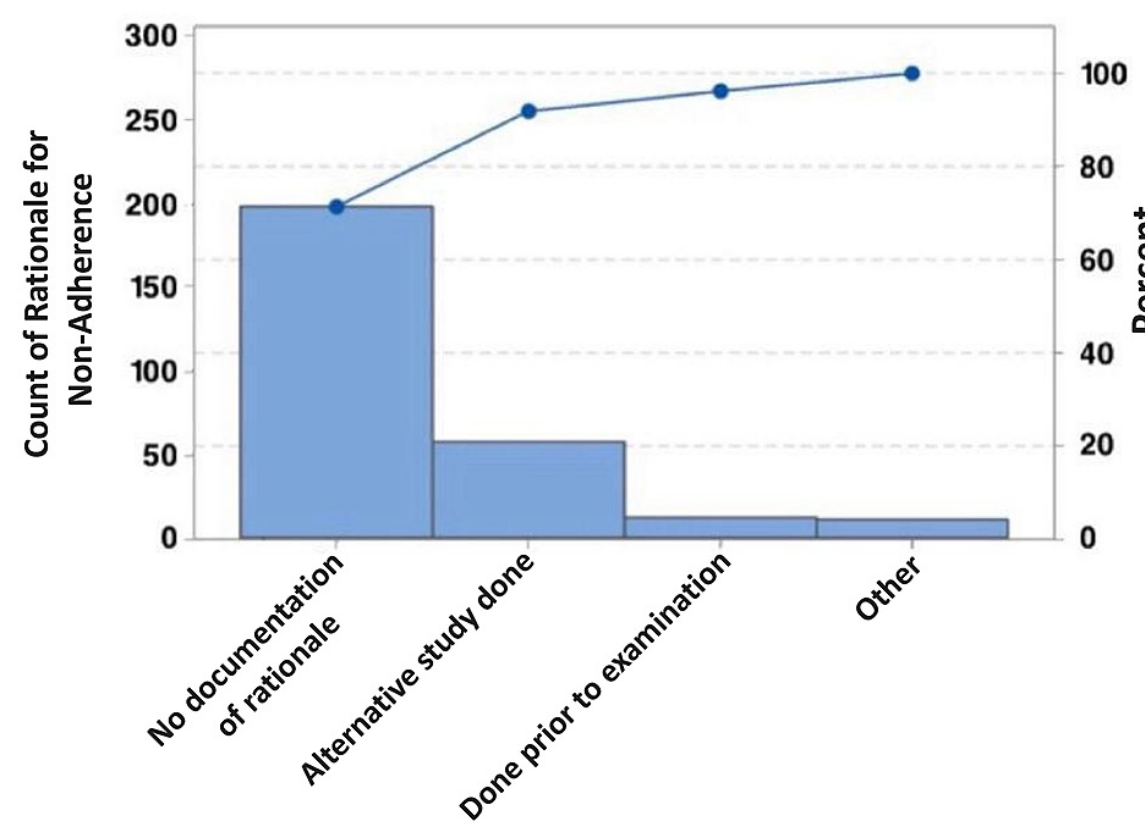

\begin{tabular}{rrrrr} 
& \multicolumn{4}{c}{ Rationale for Non-Adherence } \\
Count & 198 & 57 & 12 & 11 \\
Percent & 71.2 & 20.5 & 4.3 & 4.0 \\
Cum \% & 71.2 & 91.7 & 96.0 & 100.0
\end{tabular}

FIGURE 3: Rationale for Not Performing the Recommendation 
The recommendations that were done were compared with whether the recommendations were in the same system as the CT indication, with a likelihood ratio of $4.106(\mathrm{DF}=1, \mathrm{P}=0.043)$, indicating a significant association between the recommendations performed and whether they were performed in the same system as the CT indication. The recommendations that were done were also compared with the type of further evaluation recommended, with a likelihood ratio of $17.841(\mathrm{DF}=3, \mathrm{P}=0.001)$, indicating a significant association between the recommendations performed and the type of further evaluation recommended.

\section{Discussion}

The primary aim of the study was to assess the effectiveness of radiology report recommendations by analyzing the execution and/or response of the referring departments to the recommendations provided in the radiology report. The study findings revealed that $53.15 \%$ of the recommendations were not carried out. The secondary objective was to identify the rationale behind not following through on the recommendations. Most of the recommendations did not contain a specific rationale for why they were disregarded $(\mathrm{N}=199,71.58 \%)$.

A study at an academic children's hospital sought to determine the proportion of radiology report recommendations that were acknowledged by referring physicians [3]. The study analyzed 453 reports from 370 patients and found that 140 recommendations were not executed and only $40 \%$ were mentioned in the clinical notes. The study had a lower percentage of disregarding of recommendations compared to our study. Similarly, Intermountain Medical Center researchers found that of the 1000 CT pulmonary angiographic studies received, 9.9\% showed incidental pulmonary nodules requiring additional follow-up [11]. Only 29\% of these studies were followed up, indicating a lower rate of recommendation adherence than our study, which considered all recommendations equally. A study conducted at Staten Island University Hospital aimed to determine the number of incidental findings and the need for a follow-up of abdominal CT reports at a pediatric trauma center. The study analyzed 418 incidental findings from 345 patients and found that 60 might require outpatient monitoring. The study also concluded that approximately one-third of the patients had radiological findings not attributed to their injuries, and one-third of the patients needed further workup [12].

Another study examined whether expressions of doubt and further imaging recommendations affected the follow-up rate. The study reviewed 250 outpatient reports and found that 92 (36.8\%) lacked a timely response, whereas, in our study, $38.6 \%$ of the total recommendations were performed within six months. The study also found that further imaging recommendations had a higher chance of a lack of follow-up [13]. Another study analyzed the frequency of additional imaging recommendation acceptance. The study analyzed 430 scans from a tertiary medical center and identified further imaging recommendations in 67 cases (15.58\%). In contrast, in our study, further imaging recommendations were the majority (78.39\%). The recommendations were not completed in 43 cases (64.18\%), indicating a percentage higher than the one reported in our study. No rationale was documented in $38.6 \%$ of the recommendations not performed, whereas, in our study, $71.58 \%$ of recommendations not performed did not have a documented rationale. The study also found that, contrary to concerns of auto referral to further imaging by radiologists, the rate of these referrals was less than that of prior studies [14].

The study may have been limited by the relatively short time frame of abdominal and pelvic CT reports, from October 1, 2017, to December 31, 2017. Moreover, the study was conducted at a single tertiary care hospital. Another possible limitation may have been that the only way to document the rationale for not performing recommendations was to add notes to the patient's medical record. Some of the recommendations were scheduled for six months or longer after the CT, which affected our analysis of how timely the recommendations would have been implemented.

\section{Conclusions}

In conclusion, by a relatively slim margin, the majority of recommendations were not performed, and in most cases, there was no documented rationale for the non-performance of the recommendations. There are some limitations to following the recommendations stated in the radiology report. However, there are certain measures that can be taken to prevent the poor utility of the recommendations. The attitude of physicians toward following these recommendations as a medical obligation enhances the chances that a proper response will ensue. A possible solution method to improve the quality of radiology reports is by implementing guidelines or advocating the use of a more structured style of reporting. Verbal and electronic notification can be associated with increased follow-up of recommendations. Audit systems or key performance indicators (KPIs) can also be implemented to improve recommendation compliance.

\section{Additional Information \\ Disclosures}

Human subjects: Consent was obtained or waived by all participants in this study. The ethics committee of King Abdullah International Medical Research Center issued approval \#SP18/331/J. Animal subjects: All authors have confirmed that this study did not involve animal subjects or tissue. Conflicts of interest: In compliance with the ICMJE uniform disclosure form, all authors declare the following: Payment/services 
info: All authors have declared that no financial support was received from any organization for the submitted work. Financial relationships: All authors have declared that they have no financial relationships at present or within the previous three years with any organizations that might have an interest in the submitted work. Other relationships: All authors have declared that there are no other relationships or activities that could appear to have influenced the submitted work.

\section{References}

1. Ridley LJ: Guide to the radiology report. Australas Radiol. 2002, 46:366-9. 10.1046/j.1440-1673.2002.01084.x

2. Goergen SK, Pool FJ, Turner TJ, et al.: Evidence-based guideline for the written radiology report: methods, recommendations and implementation challenges. J Med Imaging Radiat Oncol. 2013, 57:1-7. 10.1111/1754-9485.12014

3. Lee B, Otero HJ, Whitehead MT: The fate of radiology report recommendations at a pediatric medical center. Pediatr Radiol. 2017, 47:1724-9. 10.1007/s00247-017-3960-4

4. Wallis A, McCoubrie P: The radiology report--are we getting the message across? . Clin Radiol. 2011, 66:1015-22. 10.1016/j.crad.2011.05.013

5. Schwartz LH, Panicek DM, Berk AR, Li Y, Hricak H: Improving communication of diagnostic radiology findings through structured reporting. Radiology. 2011, 260:174-81. 10.1148/radiol.11101913

6. Reiner BI: Strategies for radiology reporting and communication. Part 1: challenges and heightened expectations. J Digit Imaging. 2013, 26:610-3. 10.1007/s10278-013-9615-6

7. Reiner B: Uncovering and improving upon the inherent deficiencies of radiology reporting through data mining. J Digit Imaging. 2010, 23:109-18. 10.1007/s10278-010-9279-4

8. Wickramarachchi BN, Meyer-Rochow GY, McAnulty K, Conaglen JV, Elston MS: Adherence to adrenal incidentaloma guidelines is influenced by radiology report recommendations. ANZ J Surg. 2016, 86:483-6. 10.1111/ans.12799

9. Sloan CE, Chadalavada SC, Cook TS, Langlotz CP, Schnall MD, Zafar HM: Assessment of follow-up completeness and notification preferences for imaging findings of possible cancer: what happens after radiologists submit their reports?. Acad Radiol. 2014, 21:1579-86. 10.1016/j.acra.2014.07.006

10. Dalal S, Hombal V, Weng WH, et al.: Determining follow-up imaging study using radiology reports . J Digit Imaging. 2020, 33:121-30. 10.1007/s10278-019-00260-w

11. Blagev DP, Lloyd JF, Conner K, et al.: Follow-up of incidental pulmonary nodules and the radiology report . J Am Coll Radiol. 2014, 11:378-83. 10.1016/j.jacr.2013.08.003

12. Daoud Y, Philip A, Altberg G, Leader H, Neuman J, Hahn B: Incidental findings on pediatric abdominal computed tomography at a pediatric trauma center. J Emerg Med. 2017, 53:616-22. 10.1016/j.jemermed.2017.08.003

13. Al-Mutairi A, Meyer AN, Chang P, Singh H: Lack of timely follow-up of abnormal imaging results and radiologists' recommendations. J Am Coll Radiol. 2015, 12:385-9. 10.1016/j.jacr.2014.09.031

14. Hanley O, Lotfi A, Sanborn T, et al.: Radiologists' recommendations for additional imaging on inpatient CT studies: do referring physicians follow them?. South Med J. 2017, 110:770-4

10.14423/SMJ.0000000000000741 